REVISTA CIENCIAS BIOMÉDICAS

PRESENTACIÓN DE CASOS CLÍNICOS

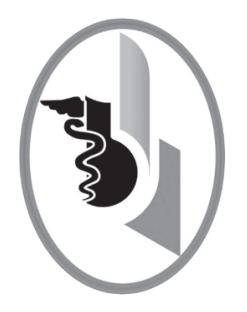

\title{
ABDOMEN AGUDO POR HERNIA DE AMYAND
}

\author{
AMYAND`S HERNIA PRESENTING AS AN ACUTE ABDOMEN
}

De la Peña López Jairo ${ }^{1}$

Silgado Kerguelen Ricardo ${ }^{1}$

Calderón Morón Hernán²

Correspondencia: jdelapenalopez@yahoo.es

Recibido para evaluación: noviembre - 02 - 2010. Aceptado para publicación: marzo - 01- 2011

\section{RESUMEN}

En el servicio de urgencia se observa con frecuencia la consulta de pacientes con cuadros abdominales de presentación aguda, que ameritan intervención quirúrgica. La apendicitis aguda es la principal patología en orden de frecuencia, sin embargo la presencia de una apendicitis aguda en un saco herniario inguinal incarcerado es poco frecuente. Esta situación clínica es conocida con el nombre de hernia de Amyand. Se presenta caso clínico de un paciente con retardo mental leve, que presentó dolor abdominal con evolución de una semana. Al consultar se evidenció cuadro relacionado con síndrome de respuesta inflamatoria sistémica y características clínicas de abdomen agudo con proceso herniario inguinal derecho incarcerado. En el acto quirúrgico se encontró peritonitis purulenta y un severo proceso adherencial. Se cumplió drenaje de la peritonitis y corrección de la hernia con técnica de Bassini. Rev.cienc.biomed. 2011; 2 (1): 106-110

\section{PALABRAS CLAVES}

Abdomen agudo. Hernia de Amyand. Hernia incarcerada. Síndrome de respuesta inflamatoria sistémica.

\section{SUMMARY}

Acute abdomen requiring surgical management is a frequent consultation at emergency department. Acute appendicitis is the most common cause, however the presence of acute appendicitis in an incarcerated inguinal hernia is rare, and this process is known as the eponymous Amyand's hernia. We report a case who presented with acute abdomen and a review of the literature. Our patient had a history of mild mental retardation; he had had clinical manifestations of abdominal pain one week earlier but family did not give relevance to it. At consultation time, he presents a systemic inflammatory response syndrome and acute abdomen with incarcerated right inguinal hernia; it was performed resuscitation with optimization of vital signs and surgical management with the combined approach, finding a severe purulent peritonitis adhesive process. Drainage is performed and correction of the hernia with Bassini technique. Rev.cienc.

biomed.2011; 2(1): 106-110

\section{KEYWORDS}

Acute abdomen, Amyand's hernia. Incarcerated hernia. Systemic inflammatory response syndrome (SIRS).

\footnotetext{
1 Médico. Estudiante de postgrado. Cirugía General. Departamento Quirúrgico. Facultad de Medicina. Universidad de Cartagena. Colombia.

2 Médico. Especialista en Cirugía General. Hospital Universitario del Caribe. Cartagena. Colombia.
} 


\section{INTRODUCCIÓN}

El abdomen agudo es uno de los problemas más frecuentes de consulta en el servicio de urgencias, e involucra la participación de diversos especialistas como cirujanos, internistas, pediatras y ginecólogos. Plantea un gran abanico de probabilidades diagnósticas, lo que exige un enfoque sistemático y ordenado, que con frecuencia implica un manejo quirúrgico (1). Dentro de las etiologías, la apendicitis aguda ocupa el primer lugar incluso en el embarazo (2). Tiene mayor incidencia entre los 10 y los 30 años de edad. Aún así no deja de ser uno de los principales diagnósticos diferenciales en el anciano con dolor abdominal (3).

La interpretación temprana del cuadro clínico de apendicitis aguda no debiera en general, presentar dificultades. Sin embargo, la amplia variabilidad de las posiciones del apéndice, sobre todo en estadios inflamatorios conlleva manifestaciones atípicas (6). Es poco frecuente la ubicación de la apéndice inflamada en el interior del saco inguinal o hernia de Amyand, cuyo diagnóstico prequirúrgico es difícil y en la mayoría de los casos se realiza es en el procedimiento quirúrgico.

\section{CASO CLÍNICO}

Paciente de 41 años de edad, de sexo masculino, residente y procedente del corregimiento de Barú de la ciudad de Cartagena de Indias, Colombia. De escaso nivel socio económico y educativo. Presenta retardo mental leve, sin antecedentes quirúrgicos o patológicos de importancia. En el interrogatorio, la información suministrada es escasa y poco fiable; se obtiene información de un familiar cercano quien reporta masa inguinal derecha de varios años de evolución, sin consultas médicas previas.

Desde la semana previa al ingreso el paciente había manifestado dolor persistente en la región inguinal derecha. En consulta externa se consideró manejo ambulatorio con analgésicos. Presentó exacerbación del cuadro doloroso 72 horas previas al ingreso. Acude a urgencia con dolor severo en región inguinal derecha, a nivel de la masa herniaria, fiebre, malestar general y anorexia. Estos síntomas agudos llevaban más de 48 horas.

Ingresa al servicio de urgencias con facies de enfermedad aguda y en malas condiciones generales y nutricionales. En la evaluación física como hallazgos de importancia se encuentra palidez marcada, deshidratación grado III, taquicardia, taquipnea y temperatura de $38.5^{\circ} \mathrm{C}$. Dolor a la palpación en los cuatro cuadrantes, con signos francos de irritación peritoneal. En la región inguinal derecha se observa y palpa masa inguino escrotal de tres centímetros, que no se logra reducir, dolorosa. Piel caliente e indurada.

Se realizan estudios que evidencian desequilibrio electrolítico, leucopenia y anemia microcítica hipocrómica. Gases arteriales indican acidemia metabólica. Se colocan: sonda nasogástrica, sonda vesical y catéter venoso central, sin complicaciones. Se ordena reposición hidroelectrolítica y preparación para manejo operatorio con el diagnóstico de hernia inguinal derecha estrangulada.
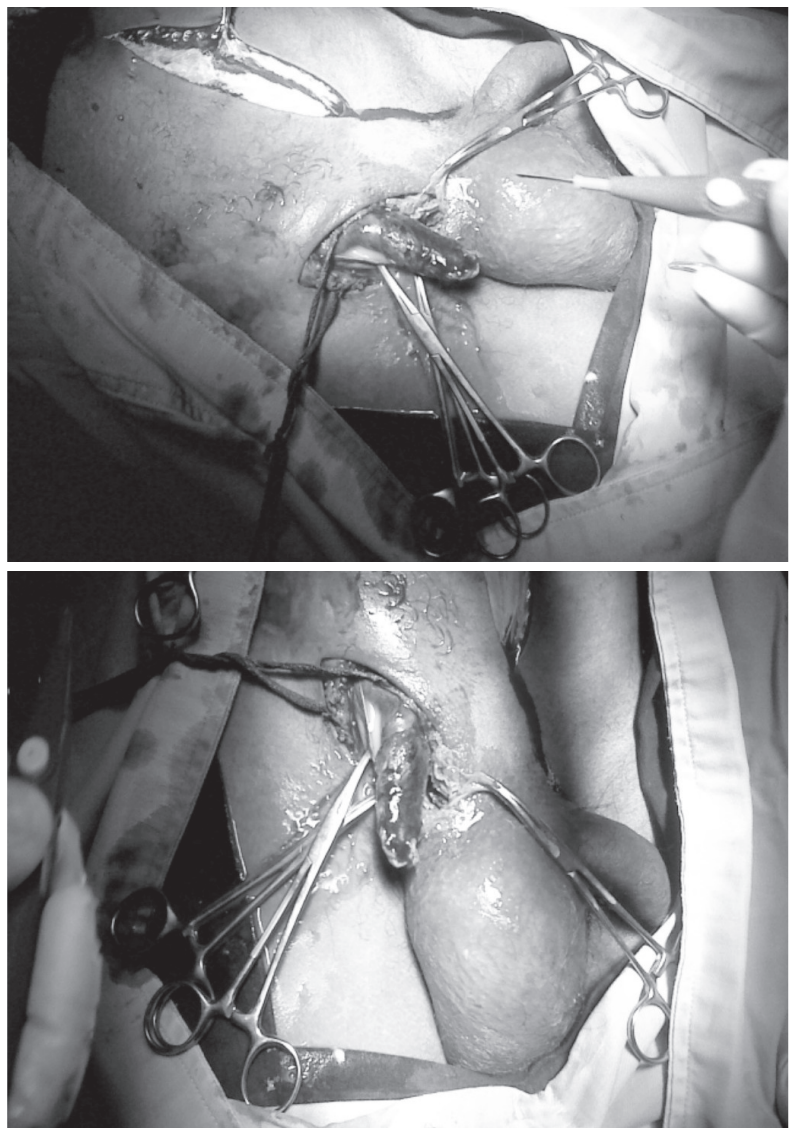
anestesia regional se aborda el canal inguinal con incisión de Marrow, observándose intenso edema del cordón alrededor de la masa incarcerada. Se diseca el saco, se realiza su apertura y se encuentra en su interior la presencia del apéndice cecal con cuerpo extraño (espina de pescado) en tercio medio y perforación en la punta. Salida de material purulento en moderada cantidad.

No se logra adecuado acceso a la base del apéndice ni del ciego, por lo que se decide realizar incisión media infraumbilical, encontrándose pus en cantidad de $300 \mathrm{~m}$. en la región pericecal y en el fondo del Saco de Douglas, del que se toma muestra para cultivo y antibiograma. Se lava la cavidad abdominal, y practica apendiceptomía por técnica de Pouchet.

\section{DISCUSIÓN}

Es infrecuente esta entidad. El apéndice se encuentra en un saco herniario en el $0.1 \%$ de los procesos inflamatorios de la misma, como lo reporta Ryan en 1937 en una serie de casos de apendicitis $(4,5)$.

En 1731 fue Garangeot quien por primera vez encontró el apéndice no inflamada en un saco herniario inguinal. En 1735 Amyand realizó al respecto la primera apendiceptomía en un niño de 11 años en el Hospital de St. George, Londres. El paciente tenía un cuadro de apendicitis aguda perforada por un alfiler, y estaba contenida en un saco herniario. Como coincidencia histórica, la primera apendiceptomía con éxito realizada por Hall en los Estados Unidos en 1886, fue precisamente en una hernia de Amyand (4, 5, 7).

Es poco frecuente la hernia de Amyand, sin embargo, se encuentran reportes que señalan que esta modalidad de hernia del lado izquierdo, se han asociado al situs inversus. Entidad también poco frecuente donde la mal rotación intestinal permite la ubicación del ciego del lado izquierdo. En asociación a un proceso herniario inguinal ipsilateral, se favorece la entrada del apéndice a dicho saco $(8,9)$. Se ha descrito la presencia del apéndice vermiforme en un saco herniario de una hernia crural (10). La evolución fisiopatológica de un proceso de apendicitis es conocida por todo cirujano, prevaleciendo desde la descripción realizada por Reginald Fitz patólogo, desde 1886 (11) hasta nuestros días y el evento fisiopatológico de la formación de un proceso herniario de tipo inguinal es también de igual conocimiento.

Es la presentación fisiopatológica de una hernia de Amyand lo que queda para discusión. Se postulan teorías o hipótesis. Dentro de las mas aceptadas está la dada por el ingreso de la apéndice al canal inguinal y la continua agresión del aumento de la presión intraabdominal, generando en esta también un proceso inflamatorio a nivel del cuello, con disminución del riego vascular más que por obstrucción de la luz por un fecalito, permitiendo la traslocación bacteriana, y la consiguiente evolución del proceso $(9,12,13)$. La presentación clínica de esta entidad es regularmente la aparición de una masa dolorosa en la región inguinal semejando un proceso incarcerado.

Se abre una discusión sobre el determinante fisiopatológico exacto de la apendicitis en el saco herniario, como evento primario o secundario; en ese orden dado por la obstrucción de la luz o teniendo en cuenta este último, generado por la mayor vulnerabilidad de la apéndice en el canal inguinal a los trastornos de flujo sanguíneo y al trauma de la misma. $(14,15,16)$

La realización del diagnóstico de la hernia de Amyand regularmente se hace en la sala de cirugía, donde se visualiza el apéndice de características inflamatorias en el saco inguinal. La presentación clínica se relaciona altamente a un proceso herniario incarcerado. Weber et al (17) en un seguimiento a cuarenta años, reportan 60 casos de hernia de Amyand, todas en hombre en las que solo en un caso se realiza el diagnóstico antes de cirugía. Otros (8) proponen que el dolor y los signos de irritación peritoneal, junto con la hernia encarcelada, deberían hacernos pensar en una apendicitis en el interior del saco herniario. López y colaboradores (18) en un artículo de revisión señala que los pródromos son los típicos de la apendicitis con dolor epigástrico, localizado en la fosa iliaca derecha y saco herniario encarcelado.

Dentro de la amplia gama diagnóstica se debe diferenciar esta entidad de procesos 
como hernia inguinal estrangulada con omentocele, hernia de Richter, tumor testicular con hemorragia, adenitis inguinal, epididimitis, adenitis inguinal, y la hernia femoral que es más frecuente en el sexo femenino $(18,19,20)$. Se reporta un caso de hernia de Amyand, con la presencia de un adenocarcinoma de apéndice que requirió posterior hemicolectomía derecha (21).

El diagnóstico previoal manejo quirúrgicoesde suma importancia y determina el pronóstico y el abordaje, lo cual permitiría la minimización de las complicaciones de la entidad, como la peritonitis difusa y la formación de abscesos secundarios a la perforación del apéndice dentro del saco e incluso tan graves como la fascitis necrotizante. La interpretación clínica temprana debe hacer sospechar del cuadro, no obstante la TAC ha demostrado alta sensibilidad y especificidad en el diagnóstico de apendicitis aguda y en la evaluación de la pared abdominal, con resultados excelentes (23). En la sospecha de un proceso inflamatorio del apéndice en un saco herniario inguinal se ha reportado la utilidad de la TAC en la visualización del órgano en dicho caso $(8,22)$. De cualquier manera la clínica determina el proceder quirúrgico y se sigue haciendo el diagnóstico en el acto operatorio, no siendo el TAC un estudio de rutina. El tiempo de evolución del proceso inflamatorio del apéndice va a relacionarse altamente con las complicaciones. La mayoría de estos pacientes son mayores de edad, a los cuales se les puede sumar otros factores, que en conjunto con el tiempo de evolución, se asocian a complicaciones como son: el grado de compromiso tisular, pobre estado nutricional, etc. Es probable que estos factores también jueguen un papel en las complicaciones graves, como la fasciitis necrotizante, e incluso la muerte del paciente. Hay otras complicaciones descritas, como el compromiso escrotal y la sepsis de origen testicular (24). La presentación clínica de una hernia de Amyand es la de una hernia inguinal estrangulada, lo cual la convierte en una emergencia quirúrgica. El retraso en el manejo operatorio de este proceso tiene una alta asociación a sepsis abdominal, shock séptico y muerte (25).

El tratamiento de elección propuesto es realizar la apendicectomía a través de una herniotomía con reparación primaria de la hernia utilizando la misma incisión (26). No obstante están descritas varias formas de acceso quirúrgico para esta patología. La vía preperitoneal es una de ellas. El abordaje preperitoneal consigue la apendicectomía en las mejores condiciones, manejo de asas incarceradas, drenaje de absceso y disminuye los riesgos de infección de la herida (27). Para este abordaje preperitoneal se proponen dos incisiones: la parainguinal propuesta por Logan (28) y la incisión mediana propuesta por Pans para el abordaje pre- peritoneal, quien también propone el uso de prótesis (malla) para la reparación de este defecto (29). Generalmente encontramos que la mayoría de los autores desaprueban el uso de prótesis, precisamente porque este tipo de injertos se asocia más con infección del sitio operatorio y sepsis (30). La vía combinada inguinal y abdominal, como fue el caso de nuestro paciente, se prefiere cuando el proceso de contaminación y la peritonitis no se pueden manejar por un solo abordaje. Se han reportado manejos laparoscópicos como el de Saggar (31). La técnica de reparación de la hernia mencionada con mayor frecuencia en la literatura es la clásica de Shouldice (5).

\section{CONCLUSIÓN}

Ante procesos herniarios inguinales en el servicio de urgencias no se debe olvidar la hernia de Amyand, por que la interpretación temprana de los síntomas, sumado a una adecuado interpretación de los pródromos de la enfermedad, permite elevar nuestro nivel de sospecha ante esta entidad, que se reporta con diagnostico transoperatorio en su mayor porcentaje. La intervención diagnostica puede ser apoyada con la tomografía, que muestra alta sensibilidad para los procesos herniarios de la pared abdominal y la apendicitis misma. Todo esto con el fin de minimizar complicaciones secundarias a diagnósticos tardíos que pueden redundar en aumento de morbilidad y mortalidad.

CONFLICTOS DE INTERÉS: ninguno que declarar.

FINANCIACIÓN: recursos propios de los autores. Estudio de laboratorio, material quirúrgico e insumos hospitalarios fueron aportados dentro la atención asistencial 


\section{REFERENCIAS BIBLIOGRÁFICAS}

1. Instituto Mexicano del Seguro Social. Guías de Práctica Clínica. Tratamiento de la Apendicitis aguda. 2009. Disponible en www.imss.gob.mx/NR/rdonlyres/...02CA.../GER_apendicitis_TX.pdf,

2. Patiño JF. Dolor abdominal agudo Guías de actuación en urgencias y emergencias, disponible en www.aibarra.org/Guias/5-2.htm, actualizadas a 05/10/2008

3. Sharp, H T. The Acute Abdomen During Pregnancy. Clinical Obstetrics and Gynecology, 2002; 45(2): 405-413

4. Ocampo JM, González A. Abdomen agudo en el anciano, Rev Colomb Cir 2006; 21(4): 266-282

5. Ryan WJ. Hernia of the vermiform appendix. Ann Surg 1937; 106: 135-139

6. Martín N, Mora, G, Stock RI, et al. Hernia de Amyand: presentación de un caso y revisión de la literatura. Rev. Chilena de Cirugía.2007; 59 (2): 142-144

7. Ferraina $P$, Oria A. Apéndice cecal. Cirugía. Michans. $5^{\circ}$ edición, 2002,

8. López P, Hernández L, López F. Hernia de Amyand: presentación de dos casos. Revista Cubana de Cirugia 2005;44(1): 1-7

9. Gupta S, Sharma R, Kaushik R. Left-sided Amyand's hernia. Singapore Med J 2005;46:424 -425.

10. Contreras R, García J, González J. et al. Hernia de Amyand del lado izquierdo: revisión de la bibliografía y comunicación de un caso. Revista de Especialidades Médico-Quirúrgicas 2008;13(2):88-92

11. Priego $\mathrm{P}$, Lobo $\mathrm{E}$, Moreno I, Et al. Fresneda apendicitis aguda en una hernia crural incarcerada: análisis de nuestra experiencia. Rev Esp Enferm Dig 2005; 97(10): 707-715

12. Mazeh H, Epelboym I, , Jill B.A.b. Et al, Tip appendicitis: clinical implications and management. The American Journal of Surgery 2009; 197: 211-215

13. Martín N, Mora, G, Stock RI, et al. Hernia de Amyand: presentación de un caso y revisión de la literatura. Rev. Chilena de Cirugía.2007; 59 (2): 142-144

14. Solecki R, Matyja A, Milanowski W. Amyand 's Hernia: a report of two cases. hernia 2003; 7: 5051.

15. Ash L, Hatem S, Motta G A, Veniero J. Amyand'shernia: a case report of prospective CT diagnosis inthe emergency department. Emergency Radiology 2006; 11: 231-232.

16. Breitenstein $\mathrm{S}$, Eisembach, Wille G, Et al. Incarcerated vermiform appendix in a left-sidedinguinal hernia. Hernia 2005; 9: 100- 102.

17. Powell JL. Historical Notes in Pelvic Surgery:Anecdotes on appendicitis. Journal of Pelvic Surgery 2003; 7: 39-41.

18. Weber RV, Hunt ZC, Oral JG. Amyand's hernia: etiologic and therapeutic implications of two complications. Surg Rounds. 1999;22:552-6.

19. López P, Hernández L, López F. Hernia de Amyand: presentación de dos casos. Rev Cubana Cir 2005;44(1): $20-24$

20. Salemis N.S, Nisotakis K, Nazos K, et al. Perforated appendix and periappendicular abscess within an inguinal hernia. Hernia 2006; 10, 6: 528-530

21. Osorio JK, Guzman-Valdivia G. Ipsilateral Amyand'sand Richter's hernia, complicated by necrosing fascitis. Hernia 2006; 10: 443-446.

22. Chia-lun Wu - Chao-chin Yu. Amyand's hernia with adenocarcinoid tumor. Hernia 2010; 14: 4: 423-425

23. Luch JS, Halpern D, Katz DS. Amyand's hernia: prospective CT diagnosis. J Comput Assist Tomogr 2000; 24:884-6.

24. Ulloa L, Camargo C, Carrillo J, Et al. Evaluacion tomografica de las hernias de la pared abdominal. Revista Colombiana de Radiologia. 2008; 19(2):2387-2396

25. Thakur A, Buchmiller T, Hiyama D, Shaw A, Atkinson J. Scrotal abscess following appendectomy. Pediatr Surg Int. 2001;17(7):569-71.

26. Kulah B, Kulacoglu IH, Oruc MT, Duzgun AP, Moran M, Ozmen MM, Coskun F (2001) Presentation and outcome of incarcerated external hernias in adults. Am J Surg 181:101-104

27. Nicola M, Mora G, Stock R, et al. Hernia de Amyand. Reporte de un caso. Revista Chilena de Cirugía 2007;59 (2): 142-144

28. Nguyen ET, Komenaka IK. Strangulated femoral hernia containing a perforated appendix. Can J Surg 2004;47:68-9.

29. Logan MT, Nottinghan JM. Amyand's hernia: a case report of an incarcerated and perforated appendix within an inguinal hernia and review of the literature. Am Surg. 2001;67(7):628-9.

30. Pans $A D$, Jacquet $\mathrm{N}$. Use of a preperitoneal prosthesis for strangulated groin hernia. $\mathrm{Br} \mathrm{J}$ Surg. 1997;84(3):310-12.

31. Torres HD, Roselló FJR, Del Campo AR, Canals PP, Weinman E S. Hernia de Amyand: presentación de un caso y revisión de la literatura. Arch Cir Gen Dig 2003;22: 1-5.

32. Saggar VR, Singh K, Sarangi R. Endoscopic Total Extraperitoneal management of Amyand 's hernia. Hernia 2004; 8: 164-165. 